\title{
Parameter estimation of wound rotor induction motors from transient measurements
}

\author{
Hengameh Kojooyan-Jafari, Lluís Monjo, Student Member, IEEE, Felipe Córcoles, Joaquín Pedra, \\ Member, IEEE
}

\begin{abstract}
A new method for determination of the steadystate equivalent circuit parameters of wound rotor induction motors using experimental starting transient measurements is presented. The algorithm data are the stator currents and voltages and mechanical speed. The algorithm uses the least-square method and motor dynamic equations in the synchronous reference frame. Moreover, an approximation of the rotor flux that improves the accuracy of the estimation method, as well as a detailed study of errors, is included. The estimation method is applied to starting transient measurement data of a $2 \mathrm{~kW}$ wound rotor induction motor, and parameter accuracy is verified by comparing steady-state torque- and current-slip curves calculated with the estimated and laboratory measured parameters.
\end{abstract}

Index Terms-Equivalent circuits, wound rotor induction motor, starting transients.

$\mathrm{P}$ ARAMETER determination of induction motors is an important topic because these machines are one of the most common loads in the grid. Estimation accuracy is crucial because of the importance of parameters in power system dynamic behavior during faults and other perturbations. Another important area is high performance ac drives, where parameter estimation is key in controller tuning. Several approaches to induction motor parameter estimation have been presented. IEEE Std-112 [1] describes the most usual method, which is based on short-circuit and no-load tests. Other methods are based on steady-state [2], frequency [3] or transient tests [4-10]. More recent and sophisticated procedures use a transient test with an extended Kalman filter [4][5]. Another possibility is to formulate a least-square minimization problem [6-11] and a non-linear least-square method [12][13]. Reference [6] gives a thorough description of the least-square method and dynamic equations in the rotor reference frame and [7] uses the stator reference frame. Other examples use the synchronous reference frame [8] and [12]. This transformation has the advantage that the variation of variables is smoother and their value is kept almost constant in steady state.

The main issue in this paper is to develop an estimation method. One major drawback of many parameter estimation procedures is the need for large currents in the locked-rotor

This research work was supported by the Spanish Ministerio de Ciencia e Innovación under Project ENE2009-10274.

H. Kojooyan, Ll. Monjo, F. Córcoles and J. Pedra are with the Department of Electrical Engineering, ETSEIB-UPC, Av. Diagonal 647, 08028 Barcelona, Spain (e-mails: kojooyan@iiau.ac.ir, lluis.monjo@upc.edu, corcoles@ee.upc.edu,pedra@ee.upc.edu). test, especially in big machines. Moreover, in some industrial applications the rotor may actually not be locked. For this reason, this paper measures the direct on-line starting transient, which is considered a part of motor operation.

The estimation procedure is adequate for wound rotor induction motors and single-cage motors (modeled with the steady-state equivalent circuit in Fig. 1), where the mechanical transient is decoupled from the electrical transient, i.e., the time constants of the electrical and mechanical transients have different orders of magnitude.

The method in this paper is based on:

- Transformed equations of the induction machine in the synchronous reference frame.

- Measurements of stator voltages and currents, and mechanical speed during the on-line starting transient.

- A smoothing process to calculate the derivatives of the above variables.

- A new approximation of the rotor flux.

- Validation by comparison of measured and calculated steady-state torque and current-slip curves.

The synchronous reference frame is useful for data preprocessing because of the slow variation of variables, as said before. The smoothing process makes measurements free of noise, and allows calculating derivatives numerically. The key of the paper is the presentation of an approximation of the rotor flux which is usually neglected in the literature despite its contribution to the accuracy of estimated parameters.

Finally, whereas in the literature the effectiveness of the estimation procedures is usually determined by comparing simulation parameters with estimated ones [2][3][6][8][9], stator current measurements with estimated ones [5][12], or both [7][13][14], this paper validates the accuracy of estimated parameters by comparing the steady-state torque- and currentslip curves calculated from the parameters with those measured in the laboratory.

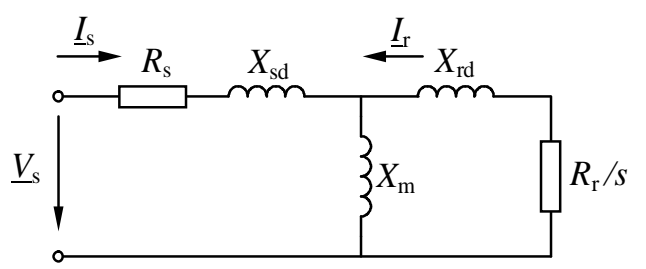

Fig. 1. Steady-state equivalent circuit for the single-cage model of the threephase induction motor. 

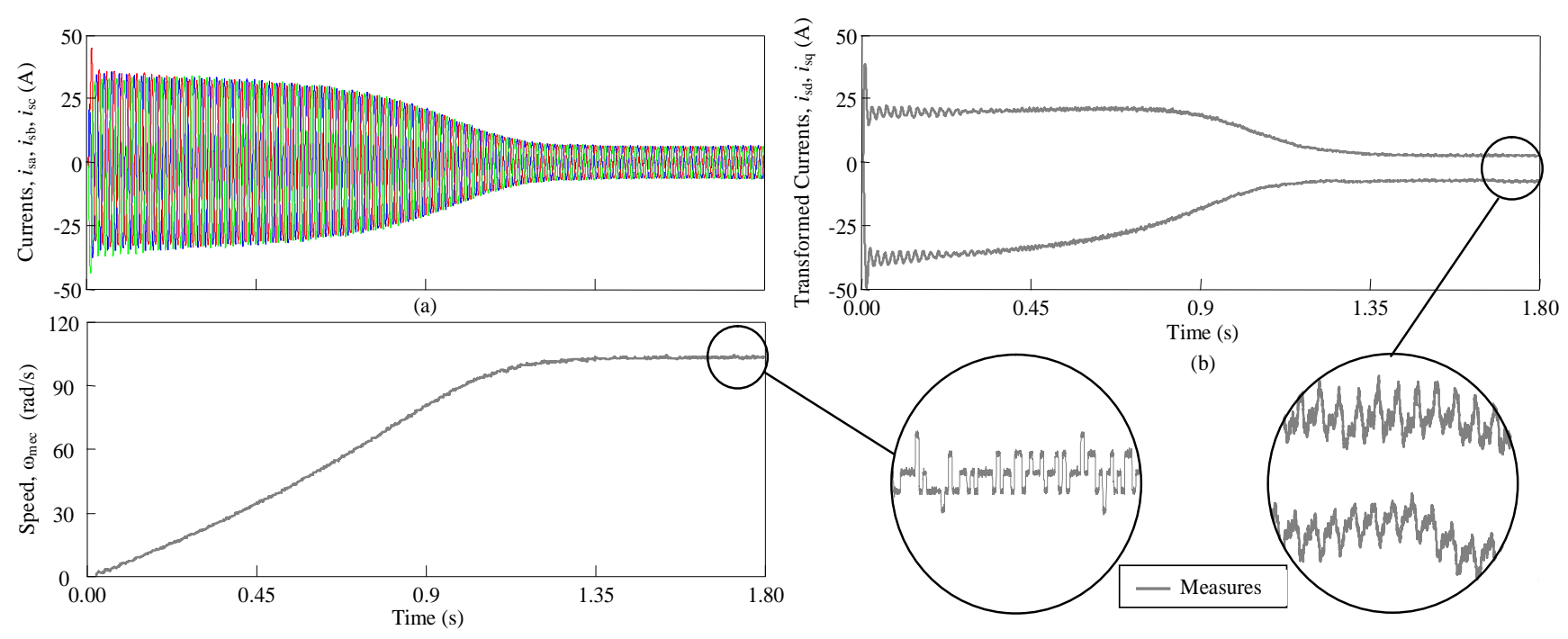

(c)

Fig. 2. Measured phase currents and mechanical speed during the starting transient of the wound rotor machine.

\section{LABORATORY MEASUREMENTS}

The wound-rotor induction motor tested in the laboratory has a $2.2 \mathrm{~kW}$ rated power, $400 \mathrm{~V}$ voltage and $50 \mathrm{~Hz}$ frequency. The laboratory setup included a programmable three-phase voltage source to supply voltage/current to the induction motor, a dc adjustable speed drive mechanically coupled to the induction motor (which adjusted the shaft speed and operated as a load), speed and current sensors and a torque transducer mounted on the shaft.

The laboratory measurements are divided into:

- Starting transient measurements under no-load conditions to estimate the parameters of the model.

- Steady-state measurements to verify the accuracy of the estimation method.

All measurements were made at three different voltages.

\section{DyNAMiC EQUATIONS}

The dynamic Park equations in the synchronous reference frame are

$$
\begin{aligned}
& v_{s d}=\left(R_{s}+L_{s} \frac{d}{d t}\right) i_{s d}-L_{s} \omega i_{s q}+M \frac{d}{d t} i_{r d}-M \omega i_{r q} \\
& v_{s q}=L_{s} \omega i_{s q}+\left(R_{s}+L_{s} \frac{d}{d t}\right) i_{s q}+M \omega i_{r d}+M \frac{d}{d t} i_{r q} \\
& 0=M \frac{d}{d t} i_{s d}-M s \omega i_{s q}+\left(R_{r}+L_{r} \frac{d}{d t}\right) i_{r d}-L_{r} s \omega i_{r q} \\
& 0=M s \omega i_{s d}+M \frac{d}{d t} i_{s q}+L_{1} s \omega i_{r d}+\left(R_{r}+L_{r} \frac{d}{d t}\right) i_{r q} \\
& T=M \cdot p\left(i_{s q} i_{r d}-i_{s d} i_{r q}\right) \quad, \quad s=\left(\omega-p \omega_{m}\right) / \omega
\end{aligned}
$$

and the $\mathrm{Ku}$ equations in the synchronous reference frame [15] are

$$
\begin{gathered}
v_{s}=\left(R_{s}+L_{s}\left(\frac{d}{d t}+j \omega\right)\right) i_{s}+M\left(\frac{d}{d t}+j \omega\right) i_{r} \\
0=M\left(\frac{d}{d t}+j s \omega\right) i_{s}+\left(R_{r}+L_{r}\left(\frac{d}{d t}+j s \omega\right)\right) i_{r} \\
T=2 p M \operatorname{Im}\left(i_{s} i_{r}^{*}\right) \quad, \quad s=\left(\omega-p \omega_{m}\right) / \omega .
\end{gathered}
$$

The relation between the equation coefficients and the steady-state equivalent circuit is

$$
\begin{aligned}
M=X_{m} / \omega ; L_{s} & =\left(X_{s d}+X_{m}\right) / \omega ; L_{r}=\left(X_{r d}+X_{m}\right) / \omega \\
L_{s d} & =L_{s}-M ; L_{r d}=L_{r}-M
\end{aligned}
$$

where $p$ is the number of pairs of poles, $\omega=2 \pi f$ is the synchronous speed and $\omega_{m}$ is the mechanical speed. The relations between Park and $\mathrm{Ku}$ variables are

$$
v_{s}=\frac{v_{s d}+j v_{s q}}{\sqrt{2}} ; \quad i_{s}=\frac{i_{s d}+j i_{s q}}{\sqrt{2}} \quad ; \quad i_{r}=\frac{i_{r d}+j i_{r q}}{\sqrt{2}}
$$

The relation between measured currents, $i_{s a}, i_{s b}, i_{s c}$, (Fig. 2a) and Park variables $i_{s d}, i_{s q}$, (Fig. $2 \mathrm{~b}$ ) in the synchronous reference frame $(\theta=\omega t)$ is

$$
\begin{aligned}
& i_{s d}=\sqrt{\frac{2}{3}}\left\{i_{s a} \cos \theta+i_{s b} \cos \left(\theta-\frac{2 \pi}{3}\right)+i_{s c} \cos \left(\theta+\frac{2 \pi}{3}\right)\right\} \\
& i_{s q}=-\sqrt{\frac{2}{3}}\left\{i_{s a} \sin \theta+i_{s b} \sin \left(\theta-\frac{2 \pi}{3}\right)+i_{s c} \sin \left(\theta+\frac{2 \pi}{3}\right)\right\}
\end{aligned}
$$

and for the voltages , $v_{s a}, v_{s b}, v_{s c}$, it is

$$
\begin{aligned}
& v_{s d}=\sqrt{\frac{2}{3}}\left\{v_{s a} \cos \theta+v_{s b} \cos \left(\theta-\frac{2 \pi}{3}\right)+v_{s c} \cos \left(\theta+\frac{2 \pi}{3}\right)\right\} \\
& v_{s q}=-\sqrt{\frac{2}{3}}\left\{v_{s a} \sin \theta+v_{s b} \sin \left(\theta-\frac{2 \pi}{3}\right)+v_{s c} \sin \left(\theta+\frac{2 \pi}{3}\right)\right\}
\end{aligned}
$$

where the voltages, $v_{s d}, v_{s q}$, are constant if the machine is fed by a purely sinusoidal voltage source. The starting transient speed is shown in Fig. 2c. 


\section{LEAST-SQUARE METHOD}

\section{A. Method equations}

Obtaining motor parameters directly from the motor dynamic equations (1) is not possible because rotor currents are generally not measurable. Hence, a new set of equations without rotor magnitudes becomes necessary. This new set, obtained in Appendix I, reads as

$$
\begin{aligned}
& K_{1} \frac{d i_{s d}}{d t}+K_{2} i_{s d}-K_{31}\left(\omega-p \omega_{m}\right) i_{s q}-K_{32} \omega i_{s q}+ \\
& -K_{4}\left(\frac{d v_{s d}}{d t}-\left(\omega-p \omega_{m}\right) v_{s q}\right)-K_{5} v_{s d}=-\frac{d^{2} i_{s d}}{d t^{2}}+ \\
& +\left(2 \omega-p \omega_{m}\right) \frac{d i_{s q}}{d t}+\omega\left(\omega-p \omega_{m}\right) i_{s d}+\frac{d p \omega_{m}}{d t} \beta \psi_{r q} \\
& K_{1} \frac{d i_{s q}}{d t}+K_{2} i_{s q}+K_{31}\left(\omega-p \omega_{m}\right) i_{s d}+K_{32} \omega i_{s d}+ \\
& -K_{4}\left(\frac{d v_{s q}}{d t}+\left(\omega-p \omega_{m}\right) v_{s d}\right)-K_{5} v_{s q}=-\frac{d^{2} i_{s q}}{d t^{2}}- \\
& -\left(2 \omega-p \omega_{m}\right) \frac{d i_{s d}}{d t}+\omega\left(\omega-p \omega_{m}\right) i_{s q}-\frac{d p \omega_{m}}{d t} \beta \psi_{r d}
\end{aligned}
$$

where

$$
\begin{aligned}
& \sigma=1-\frac{M^{2}}{L_{s} L_{r}} ; \beta=\frac{M}{\sigma L_{s} L_{r}} ; T_{r}=\frac{L_{r}}{R_{r}} ; T_{s}=\frac{L_{s}}{R_{s}} \\
& K_{1}=\frac{R_{s}}{\sigma L_{s}}+\frac{1}{\sigma T_{r}} ; K_{2}=\frac{R_{s}}{\sigma L_{s} T_{r}} ; K_{31}=\frac{1}{\sigma T_{s}} \\
& K_{32}=\frac{1}{\sigma T_{r}} ; \quad K_{4}=\frac{1}{\sigma L_{s}} ; K_{5}=\frac{1}{\sigma L_{s} T_{r}}
\end{aligned}
$$

Because rotor fluxes $\psi_{r d}, \psi_{r q}$ are not measurable, a number of studies [6-11] use equations like (7) and (8) considering the terms $\left(d p \omega_{m} / d t\right) \beta \psi_{r q}$ and $\left(d p \omega_{m} / d t\right) \beta \psi_{r d}$ negligible. This paper contributes with an approximation for these terms of the rotor fluxes. This consideration in the estimation method improves the accuracy of results, as will be demonstrated in the next Section. For this reason, Eqs. (7) and (8) are justified in Appendix I.

Using the approximation of the rotor flux, $\psi_{r}$, described in Appendix II, the following relations are obtained:

$$
\beta \psi_{r d} \approx \frac{v_{s q}}{\sigma \omega L_{s}}-i_{s d} \quad ; \quad \beta \psi_{r q} \approx-\frac{v_{s d}}{\sigma \omega L_{s}}-i_{s q}
$$

Substituting

$$
\begin{aligned}
& \frac{d p \omega_{m}}{d t} \beta \psi_{r d} \approx K_{4} \frac{d p \omega_{m}}{d t} \frac{v_{s q}}{\omega}-\frac{d p \omega_{m}}{d t} i_{s d} \\
& \frac{d p \omega_{m}}{d t} \beta \psi_{r q} \approx-K_{4} \frac{d p \omega_{m}}{d t} \frac{v_{s d}}{\omega}-\frac{d p \omega_{m}}{d t} i_{s q}
\end{aligned}
$$

in the terms of (7) and (8), we obtain a system of linear equations like

$$
\left(\begin{array}{llllll}
a_{d 1} & a_{d 2} & a_{d 3} & a_{d 4} & a_{d 5} & a_{d 6} \\
a_{q 1} & a_{q 2} & a_{q 3} & a_{q 4} & a_{q 5} & a_{q 6}
\end{array}\right)\left(\begin{array}{c}
K_{1} \\
K_{2} \\
K_{31} \\
K_{32} \\
K_{4} \\
K_{5}
\end{array}\right)=\left(\begin{array}{l}
b_{d} \\
b_{q}
\end{array}\right)
$$

where

$$
\begin{gathered}
a_{d 1}=\frac{d i_{s d}}{d t} ; a_{d 2}=i_{s d} ; a_{d 3}=-\left(\omega-p \omega_{m}\right) i_{s q} ; a_{d 4}=-\omega i_{s q} \\
a_{d 5}=-\left(\frac{d v_{s d}}{d t}-\left(\omega-p \omega_{m}\right) v_{s q}-\frac{1}{\omega} \frac{d p \omega_{m}}{d t} v_{s d}\right) \\
a_{d 6}=-v_{s d} \\
a_{q 1}=\frac{d i_{s q}}{d t} ; a_{q 2}=i_{s q} ; a_{q 3}=\left(\omega-p \omega_{m}\right) i_{s d} ; a_{q 4}=\omega i_{s d} \\
a_{q 5}=-\left(\frac{d v_{s q}}{d t}+\left(\omega-p \omega_{m}\right) v_{s d}-\frac{1}{\omega} \frac{d p \omega_{m}}{d t} v_{s q}\right) \\
a_{q 6}=-v_{s q} \\
b_{d}=-\frac{d^{2} i_{s d}}{d t^{2}}+\left(2 \omega-p \omega_{m}\right) \frac{d i_{s q}}{d t}+ \\
+\omega\left(\omega-p \omega_{m}\right) i_{s d}-\frac{d p \omega_{m}}{d t} i_{s q} \\
\quad+\frac{d^{2} i_{s q}}{d t^{2}}-\left(2 \omega-p \omega_{m}\right) \frac{d i_{s d}}{d t}+ \\
+\omega\left(\omega-p \omega_{m}\right) i_{s q}+\frac{d p \omega_{m}}{d t} i_{s d}
\end{gathered}
$$

Considering $n$ points in the study, we obtain the following over-determined linear system:

$$
\left(\begin{array}{cccccc}
a_{d 1}^{1} & a_{d 2}^{1} & a_{d 3}^{1} & a_{d 4}^{1} & a_{d 5}^{1} & a_{d 6}^{1} \\
a_{q 1}^{1} & a_{q 2}^{1} & a_{q 3}^{1} & a_{q 4}^{1} & a_{q 5}^{1} & a_{q 6}^{1} \\
a_{d 1}^{2} & a_{d 2}^{2} & a_{d 3}^{2} & a_{d 4}^{2} & a_{d 5}^{2} & a_{d 6}^{2} \\
a_{q 1}^{2} & a_{q 2}^{2} & a_{q 3}^{2} & a_{q 4}^{2} & a_{q 5}^{2} & a_{q 6}^{2} \\
\vdots & \vdots & \vdots & \vdots & \vdots & \vdots \\
a_{d 1}^{n} & a_{d 2}^{n} & a_{d 3}^{n} & a_{d 4}^{n} & a_{d 5}^{n} & a_{d 6}^{n} \\
a_{q 1}^{n} & a_{q 2}^{n} & a_{q 3}^{n} & a_{q 4}^{n} & a_{q 5}^{n} & a_{q 6}^{n}
\end{array}\right)\left(\begin{array}{c}
K_{1} \\
K_{2} \\
K_{31} \\
K_{32} \\
K_{4} \\
K_{5}
\end{array}\right)=\left(\begin{array}{c}
b_{d}^{1} \\
b_{q}^{1} \\
b_{d}^{2} \\
b_{q}^{2} \\
\vdots \\
b_{d}^{n} \\
b_{q}^{n}
\end{array}\right)
$$

which can be rewritten as

$$
\mathbf{A x}=\mathbf{b}
$$

Using the least-square regression method, the solution of this over-determined linear system is

$$
\mathbf{x}=\left(\mathbf{A}^{\mathrm{t}} \mathbf{A}\right)^{-1} \mathbf{A}^{\mathrm{t}} \mathbf{b}
$$

where $\mathbf{x}=\left(K_{1}, K_{2}, K_{31}, K_{32}, K_{4}, K_{5}\right)$.

From the parameters obtained by the least-square method, $\left(K_{1}, K_{2}, K_{31}, K_{32}, K_{4}, K_{5}\right)$, the induction motor parameters can be calculated considering the relation between parameters $L_{s d}=L_{r d}$, justified in [18]. The following relations are obtained: 


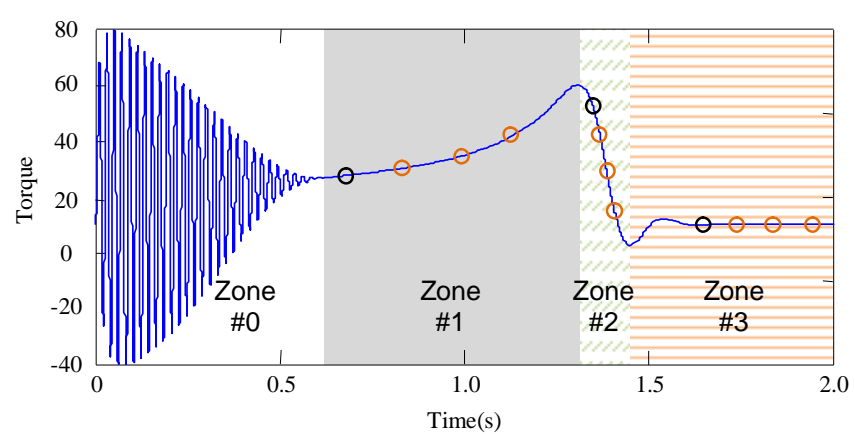

Fig. 3. Zones for the estimation method.

$$
\begin{gathered}
L_{s}=L_{r}=\frac{K_{32}}{K_{5}} ; \sigma=\frac{1}{K_{4} L_{s}} \quad ; \quad M=\sqrt{(1-\sigma) L_{s}^{2}} \\
R_{s}=\frac{L_{s}}{T_{s}}=L_{s} \sigma K_{31} \quad ; \quad R_{r}=\frac{L_{r}}{T_{r}}=L_{r} \sigma K_{32}
\end{gathered}
$$

\section{B. Application of the method}

The least-square method typically uses an excess of information. That is, it uses $n$ points to determine six parameters. To apply this method and construct the matrix defined in (16), it is necessary to have at least three points, the minimum number to find six parameters, because each point imposes two equations.

Fig. 3 illustrates the evolution of the electromagnetic torque in a starting transient. Four zones (\#0, \#1, \#2 and \#3) are delimited: \#0 is the interval where the electromagnetic transients are significant, \#1 includes points before the maximum torque, \#2 contains points between the maximum torque and the steady state, and \#3 contains points in the steady-state regime. The proposed method will be applied by choosing $n$ points in each zone from \#1 to \#3. Thus, the total number of selected points will be a minimum (or a multiple) of three (the minimum necessary to solve the system).
TABLE I

PARAMETERS OF SIMULATED MOTOR

\begin{tabular}{cccccccc}
\hline \hline$U(\mathrm{~V})$ & $R_{s}(\Omega)$ & $X_{s d}(\Omega)$ & $X_{m}(\Omega)$ & $R_{r}(\Omega)$ & $X_{r d}(\Omega)$ & $J\left(\mathrm{~kg} \cdot \mathrm{m}^{2}\right)$ & $P(\mathrm{~kW})$ \\
\hline 400 & 0.40 & 1.97 & 99.99 & 0.40 & 1.97 & 0.08 & 4.5 \\
\hline \hline
\end{tabular}

\section{SMOOTHING}

The terms in Eq. (16) use the first and second derivatives of the currents and the first derivative of the mechanical speed and voltages (13)-(15). Because measurements have noise (Fig. 2), it is difficult to obtain the derivatives numerically. To avoid this problem, a smoothing process is used to remove noise.

In order to determine the influence of smoothing on measured data and their derivatives, Simulink's SimPowerSystems Blockset simulation was implemented. Table I shows the parameters of the wound rotor induction machine in this section. Data of the three-phase voltages and currents, mechanical speed and their derivatives were obtained. As an example, Fig. 4a contains the transformed current $i_{s d}$. To make the simulation more realistic, white noise with a signal-to-noise ratio of $35 \mathrm{~dB}$ was added to the data. This noise was similar to that measured in the laboratory data of Fig. 2.

In order to smooth the current, voltage and speed measurements, a Savitzky-Golay filter was used [16][17]. This smoothing algorithm computes a moving average with coefficients determined by unweighted least-squares regression and a polynomial model of a specified degree.

In this paper, simulated and measured data are smoothed by the above method using first and second degree polynomials. Furthermore, [17] recommends to apply smoothing at least twice if a second derivative is calculated. This is done to compare derivatives of the simulated current $i_{s d}$ (Figs. $4 \mathrm{~b}$ to

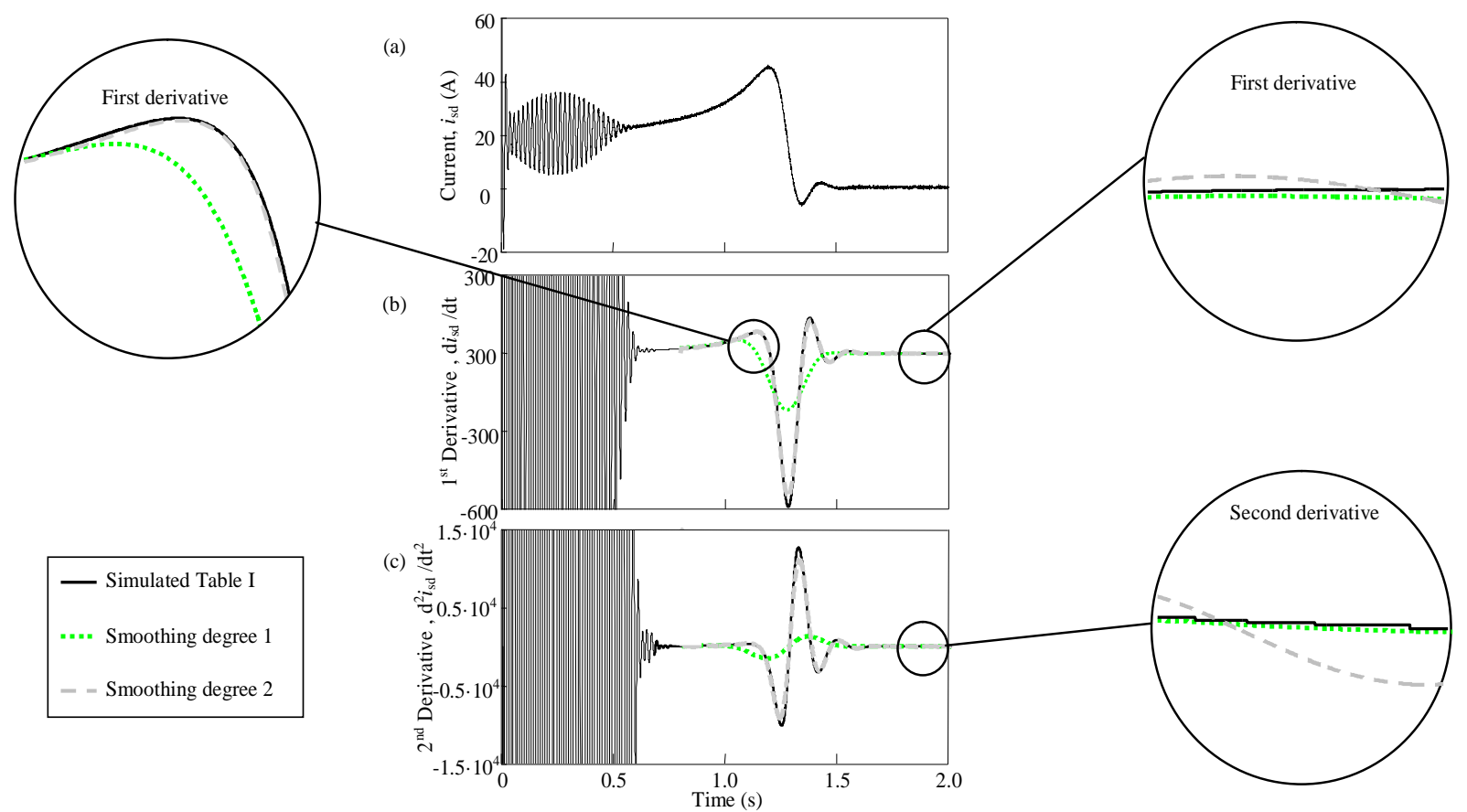

Fig. 4. (a) Simulated current $i_{s d}$ (b) First derivative of $i_{s d}$ (c) Second derivative of $i_{s d}$. 

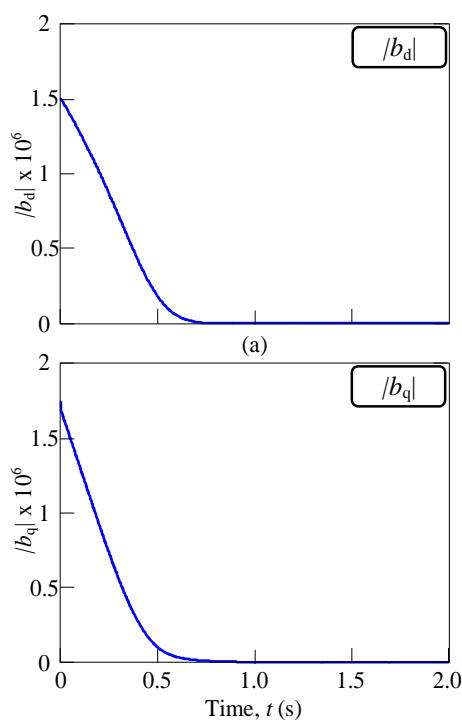

(e)
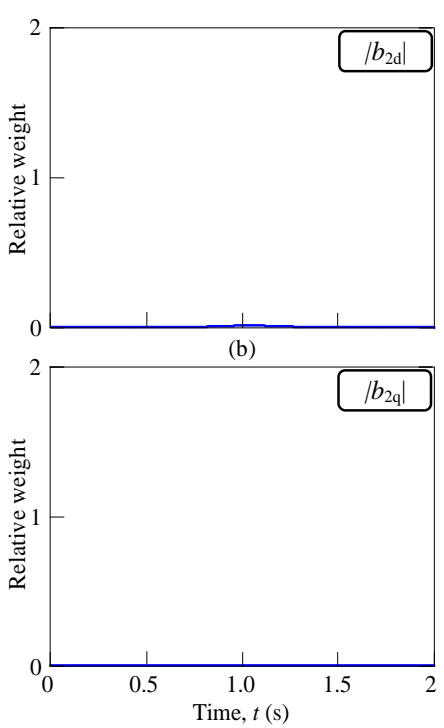

(f)
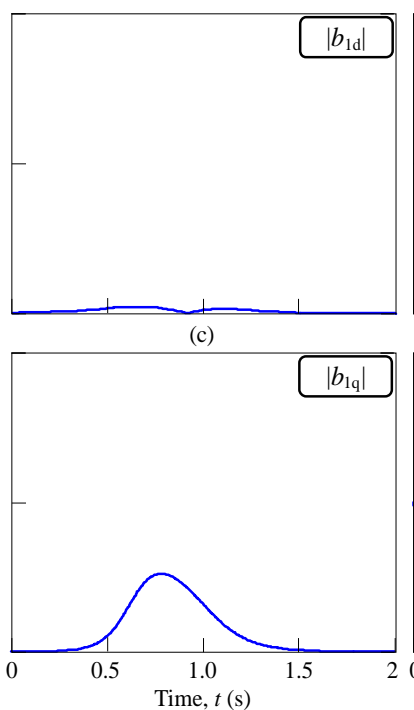

(g)
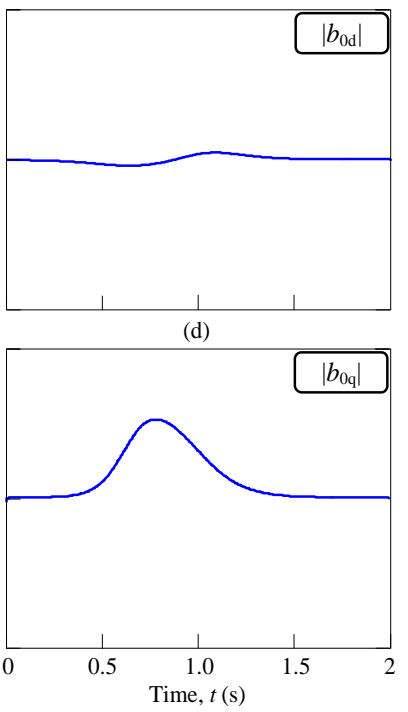

(h)

Fig. 5. Relative weight of the terms in (20) with respect to the absolute value in (15).

4d) in two different zones of the starting transient for first and second degree smoothing.

The first zoom window shows the middle of the starting transient and the second zoom window the steady-state regime. As can be seen in Figs. $4 \mathrm{~b}$ and $4 \mathrm{c}$, the real derivative (solid line) is better fitted by the first degree smoothing (dotted line) upon reaching the steady state and by the second degree smoothing (dashed line) during the mechanical transient.

In the next sections, the error caused by the numerical derivatives and smoothing is quantified.

\section{ERROR INFLUENCE ON $b_{d}$ AND $b_{q}$}

To determine the influence of the error introduced by the smoothed derivatives into coefficients $b_{d}$ and $b_{q}$ in (15), the relative weight of their summands with respect to their absolute value is evaluated. Thus, the quantities $\left|b_{2 d}\right|,\left|b_{1 d}\right|,\left|b_{0 d}\right|$, $\left|b_{2 q}\right|,\left|b_{1 q}\right|,\left|b_{0 q}\right|$ are defined as

$$
\begin{aligned}
& \left|b_{2 d}\right|=\left|\frac{d^{2} i_{s d}}{d t^{2}}\right| \frac{1}{\left|b_{d}\right|} ;\left|b_{1 d}\right|=\left|\left(2 \omega-p \omega_{m}\right) \frac{d i_{s q}}{d t}\right| \frac{1}{\left|b_{d}\right|} ; \\
& \left|b_{0 d}\right|=\left|\left(\omega\left(\omega-p \omega_{m}\right) i_{s d}-\frac{p d \omega_{m}}{d t} i_{s q}\right)\right| \frac{1}{\left|b_{d}\right|} \\
& \left|b_{2 q}\right|=\left|\frac{d^{2} i_{s q}}{d t^{2}}\right| \frac{1}{\left|b_{q}\right|} ;\left|b_{1 q}\right|=\left|\left(2 \omega-p \omega_{m}\right) \frac{d i_{s d}}{d t}\right| \frac{1}{\left|b_{q}\right|} ; \\
& \left|b_{0 q}\right|=\left|\left(\omega\left(\omega-p \omega_{m}\right) i_{s q}-\frac{p d \omega_{m}}{d t} i_{s d}\right)\right| \frac{1}{\left|b_{q}\right|}
\end{aligned}
$$

Fig. 5 illustrates the relative weight of each term in (20). From these results, it can be concluded that significant terms are those corresponding to the transformed currents, i.e., $b_{0 d}$ and $b_{0 q}$. Less important terms are those including the first derivatives, while the terms with the second derivatives are negligible. The most important implication from this study is that errors introduced by smoothing in the second derivatives hardly affect the estimation procedure.

\section{ERROR STUDY}

To verify the accuracy of computed parameters in the evaluation of machine performance, this section studies errors in depth using the motor of Table I. As the true values of the equivalent circuit parameters, (i.e., $R_{\mathrm{s}}, X_{\mathrm{sd}}, X_{\mathrm{m}}, R_{\mathrm{r}}, X_{\mathrm{rd}}$ ) are unknown, a parameter comparison cannot determine the accuracy of the estimation method [19]. Thus, the error study must focus on magnitudes derived from the steady-state machine equations correlated with machine performance, i.e., maximum torque $\left(\Gamma_{m}\right)$, starting torque $\left(\Gamma_{s}\right)$, starting current $\left(I_{s}\right)$ and no-load current $\left(I_{N L}\right)$. Therefore, the errors are defined as

$$
\begin{gathered}
\varepsilon_{\Gamma m}=\left|\frac{\Gamma_{m, e s t}-\Gamma_{m}}{\Gamma_{m}}\right| \quad ; \quad \varepsilon_{\Gamma s}=\left|\frac{\Gamma_{s, e s t}-\Gamma_{s}}{\Gamma_{s}}\right| \\
\varepsilon_{I s}=\left|\frac{I_{s, e s t}-I_{s}}{I_{s}}\right| ; \quad \varepsilon_{I N L}=\left|\frac{I_{N L, e s t}-I_{N L}}{I_{N L}}\right|
\end{gathered}
$$

As pointed out before, the estimation method needs at least three points to solve the system in (16). In the following sections, the errors are analyzed considering that the estimation procedure has one fixed point in the neighborhood of the maximum torque, another in the steady-state region, and the third moving within a range.

\section{A. Influence of $\left(d \omega_{m} / d t\right) \psi_{r}$ term}

The previous Section proposes an approximation of the rotor flux that could be considered a significant contribution because the terms affected by the rotor flux are generally neglected (as the derivative of the speed is considered negligible). 


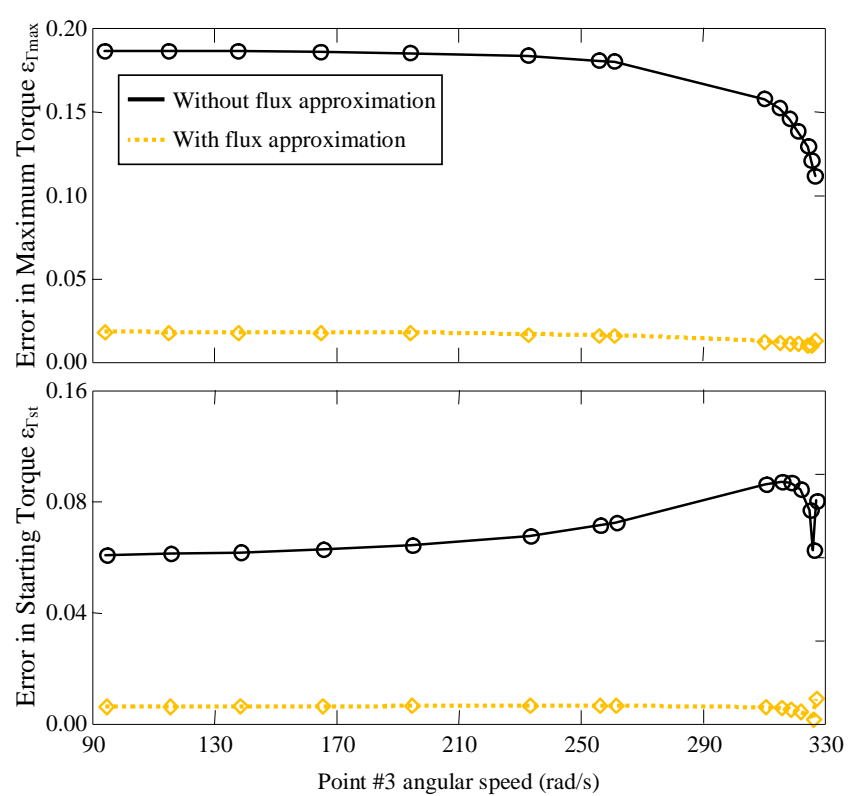

Fig. 6. Influence of the rotor flux approximation on the error in estimated maximum and starting torque.

To compare the accuracy of the estimation procedure considering or not the rotor flux approximation, Fig. 6 shows the error in the estimated maximum and starting torque for both cases. In this study, the method uses three points, i.e., two fixed points in regions \#2 and \#3 ( $\omega_{\mathrm{P} 1}=296 \mathrm{rad} / \mathrm{s}$ and $\omega_{\mathrm{P} 2}=$ $314 \mathrm{rad} / \mathrm{s})$ and a third point moving in the range $\omega_{\mathrm{P} 3}=(94$, 314) rad/s. In Fig. 6a, the line with circles represents the error when the algorithm uses the approximation that the derivative of the speed is negligible. In contrast, the line with diamonds shows the error when the algorithm uses the rotor flux approximation.

The results obtained from Fig. 6 confirm that the approximation presented in this paper is a good improvement of the estimation algorithm.

\section{B. Step size influence}

Step size affects the evaluation of the first and second derivatives, and consequently the estimation of the maximum and starting torque. The points are selected as in section V.B. Fig. 7 presents the maximum and starting torque errors. The lines with squares, diamonds and circles show the error for step sizes $h=0.1 \mathrm{~ms}, h=1 \mathrm{~ms}$ and $h=10 \mathrm{~ms}$, respectively.

From the results in Fig. 7, it can be concluded that step size has little influence on errors in derivative calculation. This is due to the use of the synchronous reference frame, where the variables vary slowly during the mechanical transient and have constant values when the steady state is reached.

\section{Influence of number of points}

In the authors' experience, better fitting is obtained with a small number of points because they can be selected near the hot regions like the maximum and steady-state torque. Additional points in each zone could provide redundant information, especially in region 3 (steady-state region), where additional data are not directly related to better results.

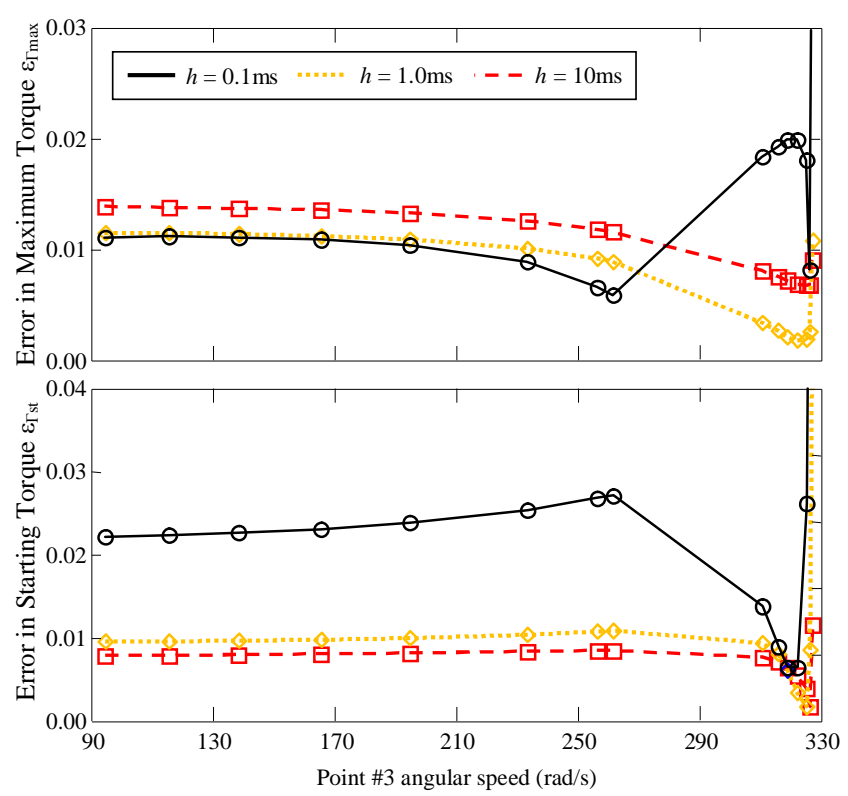

Fig. 7. Influence of the step size derivative on the error in estimated maximum and starting torque.

The best way to choose the optimal number of points for each motor is to repeat the estimation procedure (with a different number of points) and select the best suited with steady-state measurements. This is easy if the process is automatized.

Fig. 8 shows the errors between estimated and steady-state calculated maximum torque (solid line), starting torque (dotted line), starting current (dashed line) and magnetizing current (solid grey line) for a different number of points.

While using a larger number of points in parameter estimation gives reliable results, it does not increase the accuracy of the method significantly.

\section{VALIDATION OF RESULTS}

To obtain the parameters in Table II, the proposed estimation procedure was applied to the starting transient of the wound rotor induction motor described in Section 1 at different degrees of saturation (i.e., different voltages). Three cases were considered: low saturation $\left(0.25 U_{N}\right)$, medium saturation $\left(0.50 U_{N}\right)$, shown in Fig. 2, and normal saturation $\left(U_{N}\right)$. De-

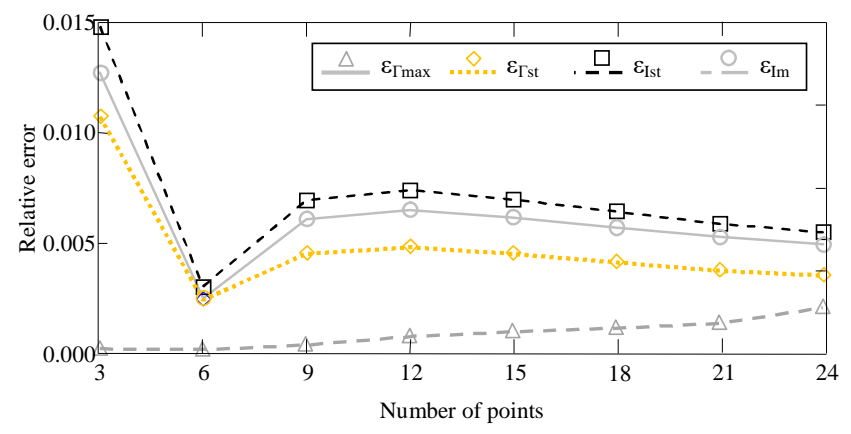

Fig. 8. Influence of equally increasing number of points in three regions on the relative error in the estimated maximum torque (solid line), starting torque (dotted line), starting current (dashed line) and magnetizing current (solid grey line). 
TABLE II

ESTIMATED PARAMETERS IN OHMS [ $\Omega$ ]

\begin{tabular}{cccccc}
\hline \hline Saturation & $R_{s}$ & $X_{s d}$ & $X_{m}$ & $R_{r}$ & $X_{r d}$ \\
\hline Low & 2.44 & 5.04 & 61.33 & 2.78 & 5.04 \\
Medium & 1.90 & 4.65 & 57.14 & 2.55 & 4.65 \\
Normal & 1.64 & 3.97 & 39.10 & 2.95 & 3.97 \\
\hline & $T_{m}(\mathrm{Nm})$ & $T_{s}(\mathrm{Nm})$ & $I_{s}(\mathrm{~A})$ & $I_{N L}(\mathrm{~A})$ & \\
\hline Low & 52.89 & 29.91 & 21.31 & 4.09 & \\
Medium & 60.48 & 34.22 & 23.21 & 3.73 & \\
Normal & 69.32 & 48.35 & 26.45 & 5.35 & \\
\hline \hline
\end{tabular}

spite the global effect of saturation on the measurements, all cases were treated as linear.

To validate the results, steady-state torque and current-slip curves were obtained with the parameters of Table II. In Fig. 9, these curves are compared with the prorated [20] to nominal voltage steady-state torque- and current-slip curves measured in the laboratory. A set of linear parameters that fit the torque- and current-slip curves were estimated from each transient at the corresponding degree of saturation. Therefore, the estimated parameters include the effect of saturation in each case.

\section{CONCLUSIONS}

A new method to estimate wound rotor and single-cage induction motor parameters from transient measurements is presented. The estimation procedure is based on the starting transient and uses Park variables in the synchronous reference frame because this formulation varies slowly during mechanical transients.

The paper also contributes a rotor flux approximation which improves the estimation procedure significantly com-
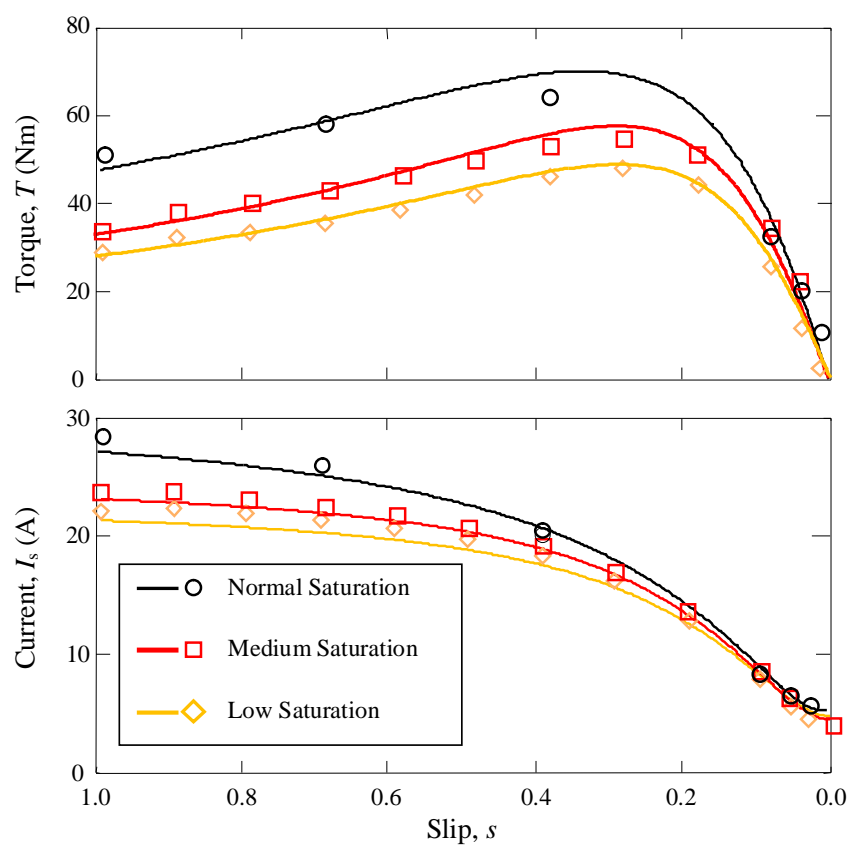

Fig. 9. Prorated measured torque- and current - slip curves for the woundrotor motor (circles) and values calculated with the proposed estimation algorithm (solid line). pared to other methods which eliminate the speed derivative term including the rotor flux. Simulation-based error studies are conducted to validate the proposed method.

Finally, the estimated parameters are used to compare the calculated torque- and current-slip curves with those from laboratory measurements. The good agreement found here contributes to the validation of the method.

\section{APPENDIX I}

By using the induction motor dynamic Ku equations in the synchronous reference frame, Eq. (2), and defining the stator and rotor fluxes as

$$
\left.\begin{array}{l}
\psi_{s}=L_{s} i_{s}+M i_{r} \\
\psi_{r}=L_{r} i_{r}+M i_{s}
\end{array}\right\} \Rightarrow i_{r}=\frac{1}{L_{r}}\left(\psi_{r}-M i_{s}\right)
$$

the rotor current is eliminated and the following equations are obtained:

$$
\begin{gathered}
v_{s}=\left(R_{s}+\left(L_{s}-\frac{M^{2}}{L_{r}}\right)\left(\frac{d}{d t}+j \omega\right)\right) i_{s}+\frac{M}{L_{r}}\left(\frac{d}{d t}+j \omega\right) \psi_{r} \\
0=-\frac{R_{r} M}{L_{r}} i_{s}+\left(\frac{R_{r}}{L_{r}}+\left(\frac{d}{d t}+j\left(\omega-p \omega_{m}\right)\right)\right) \psi_{r}
\end{gathered}
$$

These dynamic equations can be rewritten as a system of ordinary differential equations in complex variables:

$$
\left.\begin{array}{l}
\frac{d i_{s}}{d t}=(-\gamma-j \omega) i_{s}+\left(\frac{\beta}{T_{r}}-j p \omega_{m} \beta\right) \psi_{r}+\frac{1}{\sigma L_{s}} v_{s} \\
\frac{d \psi_{r}}{d t}=\frac{M}{T_{r}} i_{s}-\left(\frac{1}{T_{r}}+j\left(\omega-p \omega_{m}\right)\right) \psi_{r}
\end{array}\right\}
$$

where

$$
\begin{gathered}
\sigma=1-\frac{M^{2}}{L_{s} L_{r}} ; \beta=\frac{M}{\sigma L_{s} L_{r}} \quad ; \quad T_{r}=\frac{L_{r}}{R_{r}} \\
\gamma=\frac{R_{s} L_{r}^{2}+R_{r} M^{2}}{L_{r}\left(L_{s} L_{r}-M^{2}\right)}=\frac{R_{s}}{\sigma L_{s}}+\frac{R_{s}}{L_{s}} \frac{M^{2}}{\sigma L_{s} L_{r}}
\end{gathered}
$$

The rotor flux in (24) must be eliminated because it cannot be measured. Rewriting $i_{s}$ in $x_{1}$ and $\psi_{r}$ in $x_{2}$ results in

$$
\begin{aligned}
& \dot{x}_{1}=A x_{1}+B x_{2}+E v \\
& \dot{x}_{2}=C x_{1}+D x_{2}
\end{aligned}
$$

where the derivatives are denoted by a dot. By calculating the derivative of the first equation, we obtain

$$
\ddot{x}_{1}=A \dot{x}_{1}+B \dot{x}_{2}+\dot{B} x_{2}+E \dot{v}
$$

where coefficients $A$ and $E$ are constant. By using the equations

$$
\begin{aligned}
& x_{2}=\frac{1}{B}\left(\dot{x}_{1}-A x_{1}-E v\right) \\
& \dot{x}_{2}=C x_{1}+D x_{2}
\end{aligned}
$$

and replacing them in Eq. (27), we obtain 


$$
\begin{aligned}
& \ddot{x}_{1}=A \dot{x}_{1}+B\left(C x_{1}+D x_{2}\right)+\dot{B} x_{2}+E \dot{v}= \\
& =A \dot{x}_{1}+B\left(C x_{1}+D \frac{1}{B}\left(\dot{x}_{1}-A x_{1}-E v\right)\right)+\dot{B} x_{2}+E \dot{v}
\end{aligned}
$$

where by reordering the results, we have

$$
\ddot{x}_{1}=(A+D) \dot{x}_{1}+(B C-D A) x_{1}+\dot{B} x_{2}+E \dot{v}-D E v
$$

The literature uses the approximation $\dot{B}=0$ to eliminate $x_{2}$. This paper keeps this term and uses an approximation of $x_{2}$, i.e., the rotor flux $\psi_{r}$. Then, the coefficients in (30) are

$$
\begin{aligned}
& (A+D)=(-\gamma-j \omega)-\left(\frac{1}{T_{r}}+j\left(\omega-p \omega_{m}\right)\right)= \\
& =-j\left(2 \omega-p \omega_{m}\right)-\left(\frac{1}{T_{r}}+\gamma\right)=-j\left(2 \omega-p \omega_{m}\right)-K_{1} \\
& (B C-D A)=\left(\frac{\beta}{T_{r}}-j p \omega_{m} \beta\right) \frac{M}{T_{r}}+ \\
& +\left(\frac{1}{T_{r}}+j\left(\omega-p \omega_{m}\right)\right)(-\gamma-j \omega)=\frac{\beta M}{T_{r}^{2}}-j p \omega_{m} \frac{\beta M}{T_{r}}+ \\
& -\frac{\gamma}{T_{r}}-\frac{j \omega}{T_{r}}-j \gamma\left(\omega-p \omega_{m}\right)+\omega\left(\omega-p \omega_{m}\right)= \\
& =\left(\frac{\beta M}{T_{r}^{2}}-\frac{\gamma}{T_{r}}\right)+j p \omega_{m}\left(\gamma-\frac{\beta M}{T_{r}}\right)-j \omega\left(\frac{1}{T_{r}}+\gamma\right)+ \\
& +\omega\left(\omega-p \omega_{m}\right)= \\
& =-K_{2}-j K_{31}\left(\omega-p \omega_{m}\right)-j \omega K_{32}+\omega\left(\omega-p \omega_{m}\right) \\
& \quad(-D E)=\left(\frac{1}{T_{r}}+j\left(\omega-p \omega_{m}\right)\right) \frac{1}{\sigma L_{s}}=\frac{1}{\sigma L_{s} T_{r}}+ \\
& \quad+\frac{j}{\sigma L_{s}}\left(\omega-p \omega_{m}\right)=j K_{4}\left(\omega-p \omega_{m}\right)+K_{5} \\
& \quad E=\frac{1}{\sigma L_{s}}=K_{4} \quad ; \quad \dot{B}=-j p \beta \dot{\omega}_{m} \\
& \quad(\omega)
\end{aligned}
$$

from where the coefficients in Eq. (9) are obtained. Replacing these coefficients in Eq. (30) results in

$$
\begin{aligned}
& \frac{d^{2} i_{s}}{d t^{2}}=\left(-j\left(2 \omega-p \omega_{m}\right)-K_{1}\right) \frac{d i_{s}}{d t}+ \\
& +\left(-K_{2}-j K_{31}\left(\omega-p \omega_{m}\right)-j \omega K_{32}+\omega\left(\omega-p \omega_{m}\right)\right) i_{s}+ \\
& +K_{4}\left(\frac{d v_{s}}{d t}+j\left(\omega-p \omega_{m}\right) v_{s}\right)+K_{5} v_{s}-j \frac{d p \omega_{m}}{d t} \beta \psi_{r}
\end{aligned}
$$

Finally, by reordering the terms, we obtain

$$
\begin{aligned}
& K_{1} \frac{d i_{s}}{d t}+K_{2} i_{s}+\left(j K_{31}\left(\omega-p \omega_{m}\right)+j \omega K_{32}\right) i_{s}+ \\
& -K_{4}\left(\frac{d v_{s}}{d t}+j\left(\omega-p \omega_{m}\right) v_{s}\right)-K_{5} v_{s}=-\frac{d^{2} i_{s}}{d t^{2}}+ \\
& -j\left(2 \omega-p \omega_{m}\right) \frac{d i_{s}}{d t}+\omega\left(\omega-p \omega_{m}\right) i_{s}-j \frac{d p \omega_{m}}{d t} \beta \psi_{r}
\end{aligned}
$$

This equation, expressed in $d, q$ variables, leads to Eqs. (7) and (8).

\section{Appendix II}

The rotor flux approximation is obtained from the relation between the rotor and stator fluxes:

$$
\beta \psi_{r}=\frac{1}{\sigma L_{s}} \psi_{s}-i_{s}
$$

To demonstrate this relation, the definition of $\beta$ in (25) is used:

$$
\beta \psi_{r}+i_{s}=\frac{M}{\sigma L_{s} L_{r}} \psi_{r}+i_{s}
$$

and by using the definition of $\psi_{\mathrm{r}}$ in (22) and $\sigma$ in (25), we obtain

$$
\begin{aligned}
& \sigma L_{s} L_{r}\left(\beta \psi_{r}+i_{s}\right)=M \psi_{r}+\sigma L_{s} L_{r} i_{s}= \\
& =M\left(L_{r} i_{r}+M i_{s}\right)+\left(L_{s} L_{r}-M^{2}\right) i_{s}= \\
& =L_{r}\left(L_{s} i_{s}+M i_{r}\right)=L_{r} \psi_{s}
\end{aligned}
$$
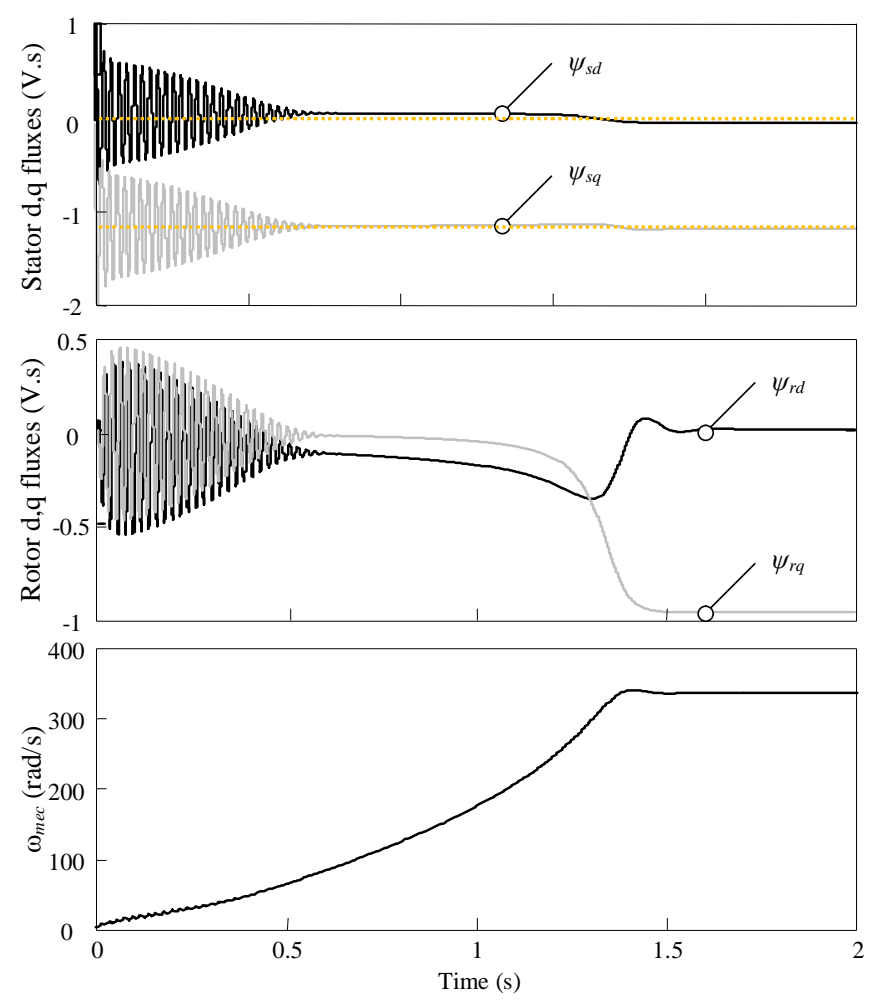

Fig. 10. Motor in Table I, (a) d and q rotor flux behavior in the starting transient, (b) d and q stator flux behavior in the starting transient, (c) motor speed evolution in the starting transient. 
Hence,

$$
\beta \psi_{r}+i_{s}=\frac{L_{r}}{\sigma L_{s} L_{r}} \psi_{s} \Rightarrow \beta \psi_{r}=\frac{1}{\sigma L_{s}} \psi_{s}-i_{s}
$$

The rotor flux approximation is obtained from the voltage stator equation

$$
v_{s}=R_{s} i_{s}+\frac{d}{d t} \psi_{s}+j \omega \psi_{s}
$$

As can be seen from the simulation in Fig. 10a, in a wound rotor induction machine, the stator flux remains almost constant after the initial electromagnetic transient. Thus, by using the approximation for the stator flux in the steady state, $v_{s} \approx j \omega \psi_{s}$, we obtain

$$
\beta \psi_{r} \approx \frac{v_{s}}{j \sigma \omega L_{s}}-i_{s}
$$

Separating (42) into $d, q$ variables results in

$$
\beta \psi_{r d} \approx \frac{v_{s q}}{\sigma \omega L_{s}}-i_{s d} \quad ; \quad \beta \psi_{r q} \approx-\frac{v_{s d}}{\sigma \omega L_{s}}-i_{s q}
$$

The above approximation is important because, as can be seen in Fig. $10 \mathrm{~b}$, the rotor fluxes, $\psi_{r d}$ and $\psi_{r q}$, vary very significantly. This variability occurs just before the mechanical speed becomes synchronous, as shown in Fig. 10c. For this reason, the accuracy of an estimation procedure based on a starting transient - such as that presented here - can be improved using the approximation in (43).

\section{REFERENCES}

[1] IEEE Standard test procedure for polyphase induction motors and generators, IEEE Std. 112-2004, Nov. 2004

[2] E. Laroche, M. Boutayeb, "Identification of the induction motor in sinusoidal mode", IEEE Trans. Energy Conversion, Vol. 25, No.1, pp. 11-19, March 2010

[3] I. Zubia, A. Zatarain, C. Alcalde, X. Ostolaza, "In situ electrical identification method for induction wind generators", IET Electric Power Applications, Vol. 5, No. 7, pp. 549-557, 2011.

[4] E. Laroche, E. Sedda, C. Durieu, "Methodological insights for online estimation of induction motor parameters", IEEE Trans. Contr. Syst. Technol., Vol. 16, No. 5, pp. 1021-1028, Sept. 2008.

[5] Lalami, A.; Wamkeue, R.; Kamwa, I.; Saad, M.; Beaudoin, J. J., "Unscented Kalman filter for non-linear estimation of induction machine parameters," Electric Power Applications, IET, vol. 6, no. 9, pp. 611-620, November 2012

[6] J. Stephan, M. Bodson, J. Chiasson, "Real-time estimation of the parameters and fluxes of induction motors", IEEE Trans. Industry Appl., Vol. 30, pp. 746-758, 1994.

[7] M. Cirrincione, M. Pucci, G. Cirrincione, G. A. Capolino, “A new experimental application of least-squares techniques for the estimation of the induction motor parameters" IEEE Trans. Industry Appl., Vol 39, pp. 1247 1256, 2003.

[8] S. R. Shaw, S. B. Leeb, "Identification of induction motor parameters from transient stator current measurements", IEEE Trans. Industrial Electronics, Vol. 46, pp. 139-149, 1999.

[9] M. Cirrincione, M. Pucci, G. Cirrincione, G. A. Capolino, "Constrained minimization for parameters estimation of induction motor in satured and unsatured conditions" IEEE Trans. Industrial Electronics, Vol 52, pp. 1391-1402, 2005.

[10] M. Cirrincione, M. Pucci, "Identification of an induction motor with the least-squares method", Elect. Eng. Res. Rep., no. 10, pp. 22-30, Dec. 2000
[11] K. Wang, J. Chiasson, M. Bodson, L. M. Tolbert, "A nonlinear leastsquares approach for identification of the induction motor parameters", IEEE Trans. Autom. Control, Vol. 50, No. 10, pp. 1622-1628, Oct. 2005.

[12] Wamkeue, R.; Aguglia, D.; Lakehal, M.; Viarouge, P., "A Two-Step Method for Identification of Nonlinear Model of Induction Machine,", IEEE Transactions on Energy Conversion, vol. 22, no. 4, pp. 801-809, Dec. 2007

[13] Wamkeue, R.; Kamawa, I.; Chacha, M., "Unbalanced Transients-Based Maximum Likelihood Identification of Induction Machine Parameters," Power Engineering Review, IEEE, vol. 22, no. 12, pp.57-57, Dec. 2002

[14] J. A. de Kock, F. S. Van der Merwe, H. J. Vermeulen, "Induction motor parameter estimation through an output error technique", IEEE Trans. Energy Conversion, Vol. 9, No.1, pp. 69-76, March 1994.

[15] Y. H. Ku, "Rotating-field theory and general analysis of synchronous and induction machines," Proc. IEE—Part IV: Instit. Monogr., vol. 99, no. 4, pp. 410-428, Dec. 1952A.

[16] Savitzky, M. J. E. Golay, "Smoothing and differentiation of data by simplified least square procedures", Anal. Chem., 36, pp. 1627-1639, 1964.

[17] T. O'Haver, "An introduction to signal processing with applications in chemical analysis",http://terpconnect.umd.edu/ toh/spectrum/TOC.html

[18] F. Corcoles, J. Pedra, M. Salichs, L. Sainz, "Analysis of the induction machine parameter identification" IEEE Trans. Energy Conversion, Vol 17, No 2, June 2002, pp. 183-190.

[19] J. Pedra, F. Corcoles, "Estimation of induction motor double-cage model parameters from manufacturer data", IEEE Trans. Energy Conversion, Vol. 19, No.2, pp. 310-317, June 2004.

[20] Ll. Monjo, F. Córcoles, and J. Pedra, "Saturation effects on torque- and current-slip curves of squirrel-cage induction motors," IEEE Trans. Energy Conversion, vol. 28, no. 2, pp. 243-254, Mar. 2013. 